\title{
Towards an Efficient, High-Fidelity Methodology for Liquid Jet Atomization Computations
}

\author{
Xiaoyi $\mathrm{Li}^{1}$, Marco Arienti ${ }^{2}$, Marios C. Soteriou ${ }^{3}$ \\ United Technologies Research Center, East Hartford, Connecticut, 06108 \\ Mark M. Sussman ${ }^{4}$ \\ Florida State University, Tallahassee, FL 32312
}

\begin{abstract}
The aim of this work is to show how adaptive mesh refinement and Lagrangian tracking can be integrated to enable high-fidelity computations of jet atomization and dispersion for industrially relevant configurations. In its present form, the Coupled Level Set and Volume of Fluid (CLSVOF) method for multiphase flow calculations is embedded in a dynamic, block-structured Adaptive Mesh Refinement (AMR) data structure which maximizes grid density at the liquid-gas interface. With the treatment proposed here, small liquid structures formed by atomization can be removed from the Eulerian description, transformed into Lagrangian particles, and advected using, for instance, a simple spherical model. As a result, mesh refinement is not required in the dilute spray region and can be contained to a smaller portion of the computational domain with minimal loss of accuracy. Two validation studies of liquid jet atomization by crossflowing gas and by jet-on-jet impingement are presented to demonstrate this new approach.
\end{abstract}

\section{Nomenclature}

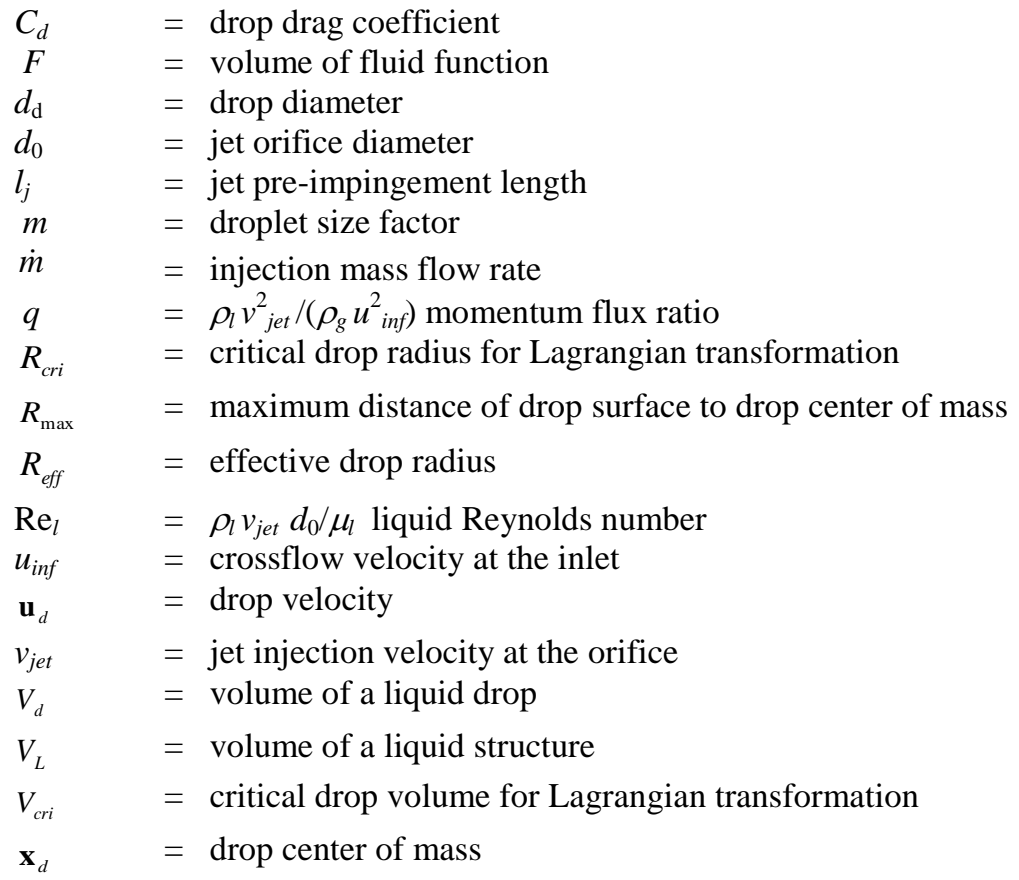

\footnotetext{
${ }^{1}$ Senior Engineer, AIAA member

${ }^{2}$ Staff Engineer, AIAA member: arientm@utrc.utc.com

${ }^{3}$ Fellow of Research, Senior AIAA member

${ }^{4}$ Associate Professor, Department of Mathematics
} 


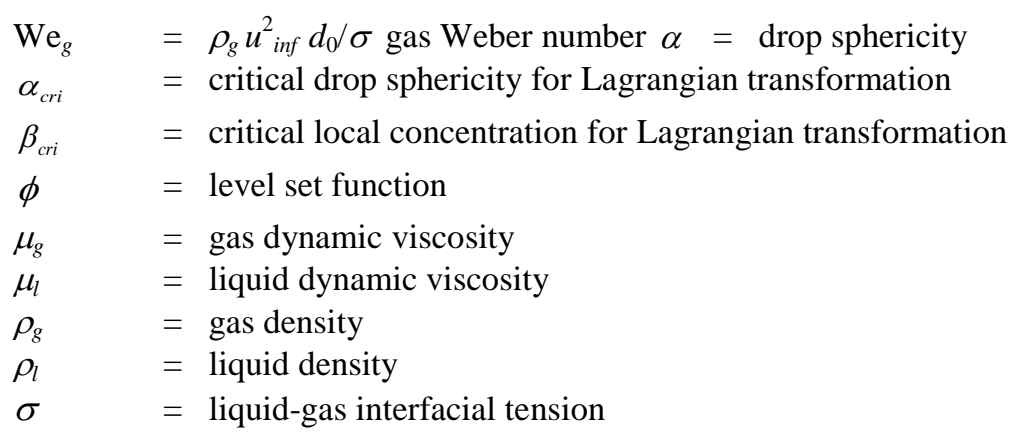

\section{Introduction}

A tomization of fuel jets and mixing of liquid propellants play a critical role in the combustion processes of aerospace engines. Accessibility to the complex near-field flow of industrially relevant atomization processes is still denied to most experiments, and high-fidelity numerical simulations have emerged as an alternative approach to gain physical insight of the injection and atomization process. Methods to directly track or capture the liquid-gas interface deformation and its topological changes include level-set (LS) ${ }^{1}$, volume-of-fluid (VOF) ${ }^{2}$ and front-tracking $(\mathrm{FT})^{3}$, as well as their descendents, such as the refined level-set grid (RLSG) method ${ }^{4}$, the Hybrid LSM \& Mars (HLSM) method ${ }^{5}$, and the coupled level-set and volume-of-fluid (CLSVOF) method ${ }^{6}$. The CLSVOF method, used in this work, takes advantages of both the accurate geometric interface representation in level set methods and the volume-preserving properties in volume of fluid methods.

Even with continuously growing computational resources, high-fidelity multiphase calculations are confronted with the broad range of spatial and temporal scales that emerge in the flow. For instance, in liquid atomization a liquid ligament can measure only a few microns in diameter just before pinch-off, whereas the scale of injectors or combustors is several orders of magnitude larger. Furthermore, on a fixed (Eulerian) grid, the multitude of droplets that is generated after atomization requires a non-negligible grid density for the droplets to be correctly advected ${ }^{7}$. A common practice is to let those droplets disappear when their size falls below the minimum grid spacing allowed by the resources at hand. This practice is not acceptable when, for instance, combustion performance needs to be assessed based on the fuel/air distribution caused by the spray.

In this respect, it can be argued that it is preferable to change the way the liquid phase is discretized in dilute spray regions, where droplets are well equilibrated with the gas flow and unlikely to collide with each other. Under the framework of the RLSG method, Herrmann ${ }^{8}$ recently implemented an algorithm that transforms small drops (small with respect to, say, the injector orifice diameter) into point-like Lagrangian particles without resolving their flow details. High-fidelity simulations of the atomization of turbulent diesel jet ${ }^{8}$ and liquid jet in cross flow ${ }^{9}$ became $^{2}$ feasible with this simplification. While convenient, it should be remembered that the representation of a droplet as a pointlike source is not completely immune from inconsistencies, particularly when thermal energy and mass exchanges between the two phases arise in addition to exchanges in momentum ${ }^{10}$. One well-known result, for instance, is that for a droplet evaporating in a stagnant or a convecting stream, the error associated with the computed evaporation rate increases as the grid size is reduced to the order of the droplet size ${ }^{11}$.

At the same time, since the development of block-structured adaptive mesh refinement (AMR) techniques for efficiently solving hyperbolic conservation laws ${ }^{12,13}$, there have been significant efforts to solve incompressible or weakly compressible flows adaptively ${ }^{14}$. AMR is now mature enough to be used for large-scale three-dimensional fluid dynamic calculations on a routine basis. 


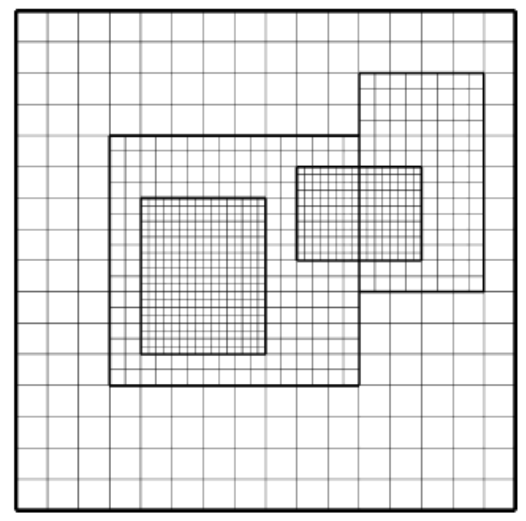

Figure 1. Diagram of the multi-level grid used in block-structured Adaptive Mesh Refinement.

The approach proposed in this paper consists in transforming the droplets in dilute spray regions into Lagrangian particles within the block-structured AMR environment. Because such droplets are removed from the Eulerian description, in the region interested by the transformation the hierarchy of refinement levels quickly reverts to the underlying base level. Thus, the grid refinement remains localized to a small volume around the jet, and the simulation cost can be eased by a relatively coarse grid far from injection. The removal of liquid structures from the Eulerian description is based on criteria of droplet size, sphericity and local droplet concentration, as explained in the next Section. The Lagrangian trajectories of the particles are tracked with the base grid flow velocity using a simple dispersed phase model.

The algorithms for the Eulerian-to-Lagrangian transformation are described in Section II and demonstrated in Section III with two examples of atomization, by gas crossflow and by jet-on-jet impingement. The primary breakup of a liquid jet in crossflow proceeds through the fragmentation of the liquid column into ligaments/large drops and through the stripping of small droplets from column surface. Different modes of surface waves due to turbulence, capillary and aerodynamic forces play critical role in the formation of ligaments and drops. Reviews of jet breakup studies can be found in Refs. 15 and 16, and more recently numerical results have begun to appear ${ }^{9,17,18}$. Atomization by jet-on-jet impingement is relevant in sub-critical conditions, such as might occur in upper stage rockets or in attitude control thrusters, where fuel and oxidizer break up and disperse with mechanisms that may not be dissimilar from the ones appearing in liquid jet in crossflow. A limited number of experiments exist in this area $^{19,20,21}$, and even fewer numerical studies ${ }^{5}$. The two demonstrations presented here include validations based on experiments at the same conditions. A summary is provided in Section IV.

\section{Numerical implementation}

The description of CLSVOF and of the dynamic mesh refinement algorithm can be found elsewhere ${ }^{6}$, together with several validation studies. Briefly, the Navier-Stokes equations for incompressible flow of two immiscible fluids (such as liquid and gas) are written in terms of a smooth level set function $\phi$, whose zero level represents the time-evolving interface. In addition to the evolution equation for $\phi$, the transport equation for the cell liquid volume fraction (the volume-of-fluid function, $F$ ) is solved. The normals used in the VOF reconstruction step are determined from the level set function. The volume fractions are then used with the normals to construct a volume preserving distance function $\phi$. In this way, volume is preserved by implementing a "local" mass fix at every iteration. Second-order accurate curvature is calculated from $F$ by the method of height fraction. A single-fluid approach is adopted, that is, properties of density and dynamic viscosity are function of $\phi$. Finally, velocity extrapolation based on $\phi$ from the liquid phase is used to approach the solution of the corresponding one-phase method in the limit of uniform vapor pressure at large liquid-to-gas density ratios.

When the adaptive mesh refinement is active, cells that are crossed by the liquid-gas interface are tagged for refinement. Starting from the base level, boxes (with a minimum size of, say, $32^{3}$ cells) are combined to cover all the tagged cells within assigned coverage efficiency (Fig. 1). This set of blocks with the same grid spacing forms level 
1. This level is in turn tagged for refinement at the interface, and the process is repeated until the required grid resolution is achieved. During the simulation, the data on the fine level are either copied from a previous time step or, when the grid structure has changed locally, conservatively interpolated from the underlying coarse level. The interface, however, is always embedded in the finest grid level to avoid gross interpolation errors. In a time step, the calculation is carried out on all levels, and the updated data on a fine level are averaged to the underlying coarser one. For a given minimum grid spacing, a higher granularity of the coverage (i.e., the prescription of many small AMR boxes) minimizes the grid count but maximizes data communication between boxes, and vice-versa. Assuming the optimal coverage closely follow the interface, it is reasonable to estimate that doubling the grid density in three dimensions corresponds to increasing the storage and the computational time by a $2^{3}$ factor instead of a $2^{4}$ factor (three dimensions plus time) for a grid without AMR. This 50\% saving in resources is repeated at every new refinement level.

\section{A. Identification of candidate liquid structures using neighbor search algorithms}

To find candidate droplets for the Lagrangian description, liquid structures separated by the gas phase need to be indentified first. Since mesh refinement is based on the location of the liquid-gas interface, small droplets are always resolved by the finest refinement level, so the identification procedure described below is performed exclusively on the finest level.

The identification uses a neighbor searching algorithm as shown in the two-dimensional example in Fig. 2. First, all the cells are tagged: $G=1$ (color gray) in the liquid phase, including the liquid-gas interface; and $G=0$ (color white) in the gas phase. Then contiguous cells are collected into separate cell lists to represent separate liquid structures. This is achieved by searching cells in the domain until a cell with $G=1$ is found. Then the cell tag is set to $G=2$ (color blue) and a new list is prepared for the liquid structure containing that first cell. Next, the immediate neighbors of the cell are searched. If a cell with $G=1$ is found, its tag is set to $G=2$ and it is added to the list of cells. The searching is carried out in the way of a multi-branch tree. The arrows in Fig. 2 indicate the direction of searching for the first few search steps. Eventually the searching is stopped when none of the neighbors of the cell within the list can be found with a tag $G=1$, and thus we obtain one isolated liquid structure represented by a list of cell indices. We continue searching for other isolated liquid structures and represent them by separate lists of cells.

As the search is carried out, running sums of the volume, the center of mass and the average velocity of the liquid structure are also updated. Note that the liquid-gas interfaces are contained in the obtained liquid structures (Fig. 2) and that the computation of the total liquid volume based on cell volume fraction is accurate. The updated liquid volume, center of mass and velocity are used to determine the eligibility of the liquid structure to be transformed into a Lagrangian particle, as described next. For instance, the liquid structure can be disqualified early if the running sum of volume is greater than a user-defined critical volume. Then no further operation, except for searching neighbors and tagging, is performed for the remaining cells. Such procedure greatly reduces the algorithm cost when searching for liquid structures with large volumes.

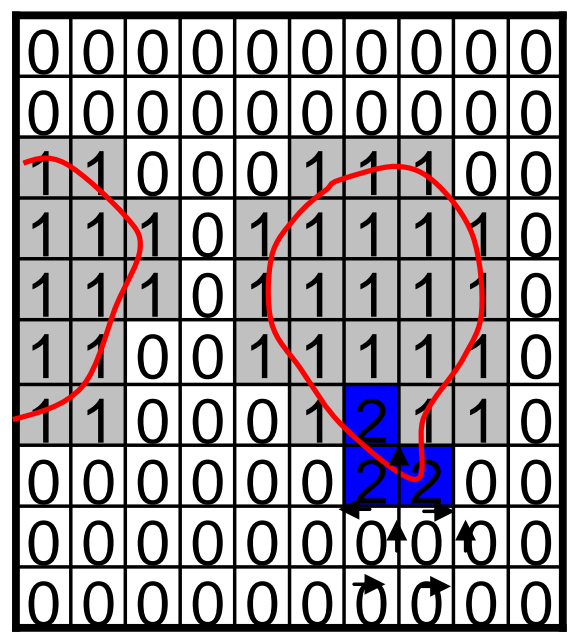

Figure 2. Two-dimensional schematics of the neighbor search algorithm for liquid structure identification. 
In Herrmann's approach ${ }^{8}$, a complex multi-block identification procedure is adopted to resolve the issue of liquid structures being separated by different blocks due to domain decomposition for parallel computing. Liquid structures are identified independently in each block and then merged together by assigning a unique identifier. We avoid this complexity by making use of the dynamic features of block-structured AMR. Particularly, any liquid structure that is cut by the boundary of boxes (blocks) is disqualified, and only the structures that are completely contained by a box are considered for transformation. This might seem arbitrary, but in reality mesh refinement needs to be performed adaptively at every time-step, so that a disqualified liquid structure has a very good chance to be completely enclosed by a new box within a few iterations. The parallelization of the identification procedure when neighboring boxes are on different processors is therefore significantly simplified, since there is no communication of liquid structure information between boxes.

\section{B. Removal of droplets from the Eulerian description}

The eligibility of the candidate liquid structures to be transformed into a Lagrangian particle is determined using numerical and physical criteria. Note that when the dimension of a fluid structure is comparable to grid spacing, numerical and reconstruction errors can become significant, with the limit situation of isolated subgrid structures being removed altogether from the calculation. A severe advection error, for instance, is observed for VOF with least squares interface reconstruction ${ }^{7}$ when the size of the liquid structure is twice the grid spacing or less. In the implementation proposed here, the volume of a candidate liquid structure is constrained to be less or equal to a critical value,

$$
V_{L} \leq V_{c r i}=\frac{4}{3} \pi R_{c r i}^{3},
$$

where $R_{c r i}=m \Delta \mathrm{x}$ and $m$ is a user-prescribed small integer ( $m=4$ for the simulation results shown in this paper). If the droplet is small enough, further breakup (referred to as secondary breakup) cannot occur due to the high surface tension compared to the aerodynamic force. In the calculations, the Weber number of the Lagrangian particles is monitored at each time step, and this diagnostics confirms the absence of the conditions for secondary breakup.

A second criterion is the droplet sphericity, defined as:

$$
\alpha=\frac{R_{\max }}{\max \left[\Delta x,\left(\frac{3}{4 \pi} V_{L}\right)^{1 / 3}\right]},
$$

where $R_{\max }$ is the maximum distance to center of mass for the liquid structure and $V_{L}$ is the volume of the liquid structure. Largely deformed liquid blobs are more probable to experience further break-up, which should be captured in the Eulerian representation. So we ensure that the transformation can occur only when $\alpha \leq \alpha_{c r i}$, where the critical value is typically $\alpha_{c r i}=4$. If $R_{\text {eff }}$ is the effective radius corresponding to the volume $V_{L}$, then $R_{\max }$ $\leq 4 \square \square \square(\Delta x, \square$ eff $)$.

We also impose a constraint of maximum local concentration $\beta_{c r i}$ of droplet below which the transformation to the Lagrangian phase can occur. This parameter separates the dense spray region, where droplet interaction can be captured in the Eulerian description, from the dilute spray region, where drop collision is assumed not to happen. The local concentration is defined as ratio of the total liquid volume in an AMR box to the volume of the box. For the cases examined in the following Section, we observed limited sensitivity of the results to variations of this parameter if $\beta_{c r i} \sim 0.01$.

At the end of this selection process, the liquid structure that meets all the above criteria is removed from the Eulerian representation by setting the level set $\phi$ and volume fraction $F$ for the cells there as

$$
\phi=-|\phi| \text { and } F=0 .
$$

While this operation is performed, the droplet volume, center of mass and velocity are calculated as

$$
V_{d}=\int_{d} d x, \quad \mathbf{x}_{d}=\frac{1}{V_{d}} \int_{d} \mathbf{x} d x, \quad \mathbf{u}_{d}=\frac{1}{V_{d}} \int_{d} \mathbf{u} d x
$$


and stored in a particle list.

\section{Lagrangian transport}

Droplet transport is calculated by solving the differential equations

$$
\frac{d \mathbf{u}_{d}}{d t}=F_{d}\left(\mathbf{u}-\mathbf{u}_{d}\right) \quad \text { and } \quad \frac{d \mathbf{x}_{d}}{d t}=\mathbf{u}_{d} .
$$

for any element of the droplet list. The drag is calculated for a rigid sphere of diameter $d_{d}$,

$$
F_{d}=\frac{18 \mu_{g}}{\rho_{l} d_{d}^{2}} \frac{C_{D} \operatorname{Re}_{d}}{24},
$$

with drag coefficient $C_{D}=0.424$ for $\operatorname{Re}_{d}=\rho_{g}\left|\mathbf{u}-\mathbf{u}_{d}\right| d_{p} / \mu_{g}>1000$ and

$$
C_{D}=\frac{24}{\operatorname{Re}_{d}}\left(1+\frac{1}{6} \operatorname{Re}_{d}^{2 / 3}\right)
$$

otherwise $^{22}$. The underlying gas flow velocity $\mathbf{u}$ is obtained by interpolation at the instantaneous droplet location. In the current implementation the Lagrangian droplets are one-way coupled to the flow so that the gas phase is not impacted by their motion.

A simple test of size and sphericity criteria is shown in Figure 3 to demonstrate how the small droplets are properly identified and removed. We apply either the size, or the sphericity, or both criteria to identify the liquid structure to be transformed, and show the remaining liquid phase after transformation under the different criteria. In frame (c) only the size restriction is enforced. Compared to frame (a) where no transformation is performed, a large number of small ligaments are removed from the Eulerian description irrespective of their shapes. In frame (d), only the sphericity restriction is enforced. Much of the highly deformed ligaments remain intact because of their stretched shape. The jet column, however, meets the sphericity criteria and therefore is removed from the Eulerian description. The correct situation is when both size and sphericity restrictions are enforced, as in frame (b). Only small and nearly spherical droplets away from the jet column are transformed. This result indicates the importance of applying both criteria of sphericity and size to avoid erroneous transformation.

Next, we verify the transformation algorithm and Lagrangian transport model by comparing the simulation of liquid jet in crossflow with Eulerian-to-Lagrangian transformation with the case without transformation. In Figure 4, the simulation without transformation (a) is compared with the case using transformation (b). Before the switch, the CLSVOF simulation was carried out for 1000 time steps. The transformation was introduced after that point only for case (b), and both cases were continued for 100 steps. The positions of the Lagrangian particles after 100 time steps match the positions of the drops in (a), indicating the accuracy of the transformation and the adequacy of the Lagrangian transport model. The grid count is significantly reduced, as shown in Figure 5, compared to the case where all droplets are resolved using all the refinement levels. In this transient phase, the cost of the simulation without transformation continues to increase due to newly created droplets. Without the conversion of droplets into particles, AMR boxes would eventually cover a substantial portion of the domain, canceling any computational advantage of adaptive mesh refinement. 

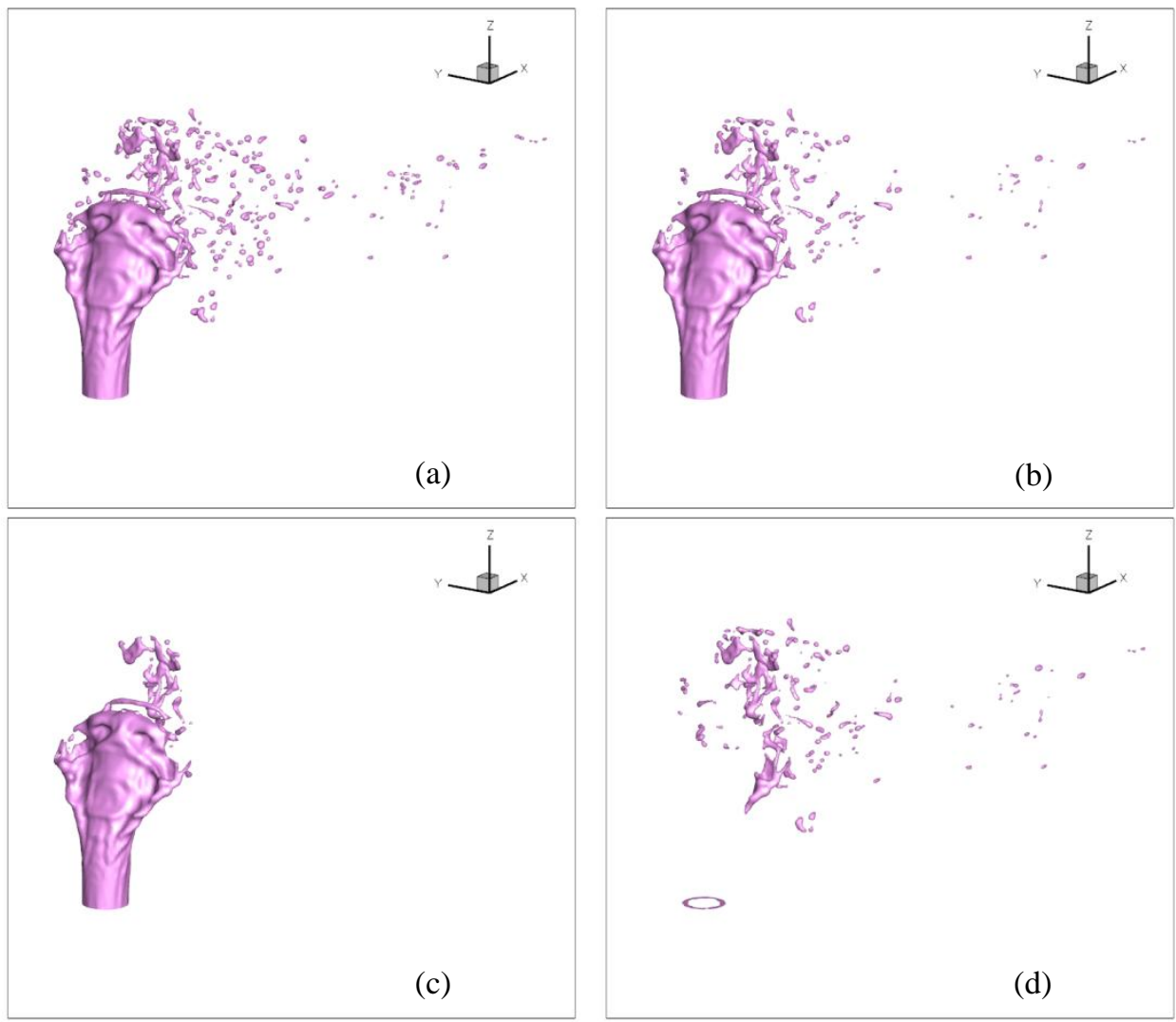

Figure 3. Demonstration of the droplet transformation criteria; (a) No transformation, (b) $R_{c r i}=5 \Delta x$ and $\alpha_{c r i}$ $=3$, (c) $R_{c r i}=5 \Delta x$ only, (d) $\alpha_{c r i}=3$ only.

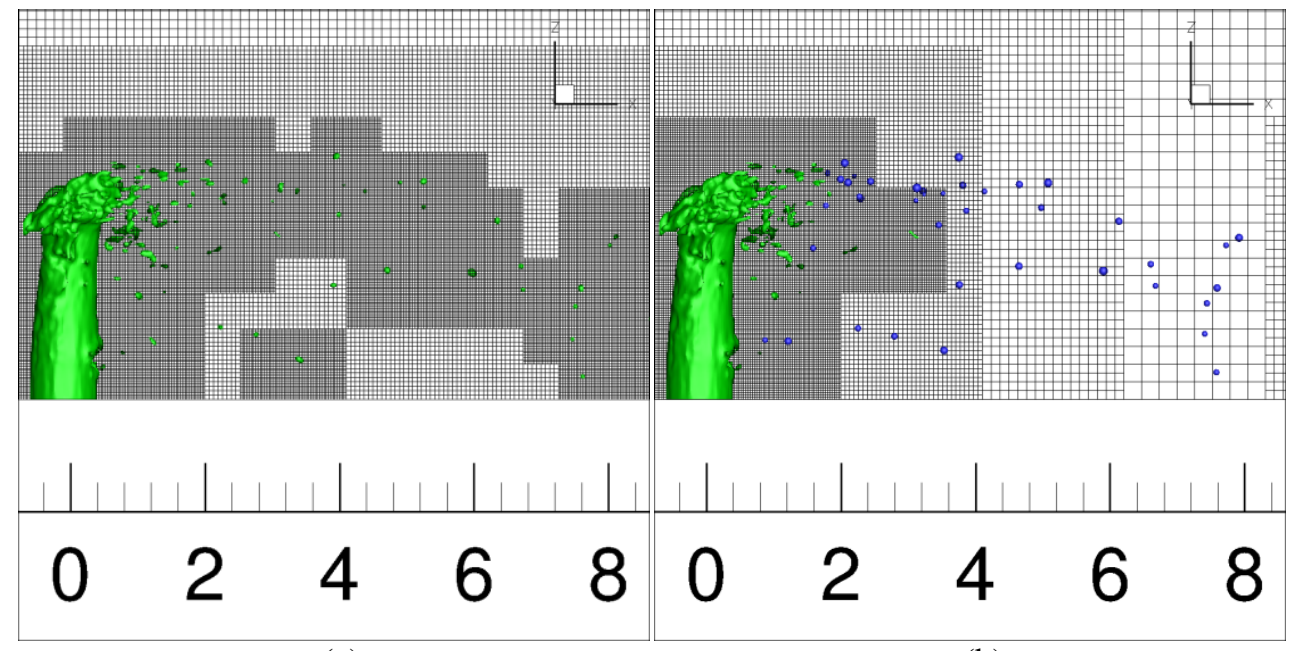

(a)

(b)

Figure 4. Snapshot of LJIC simulation after 1100 time steps without Eulerian-Lagrangian transformation (a) and with the transformation (b). 


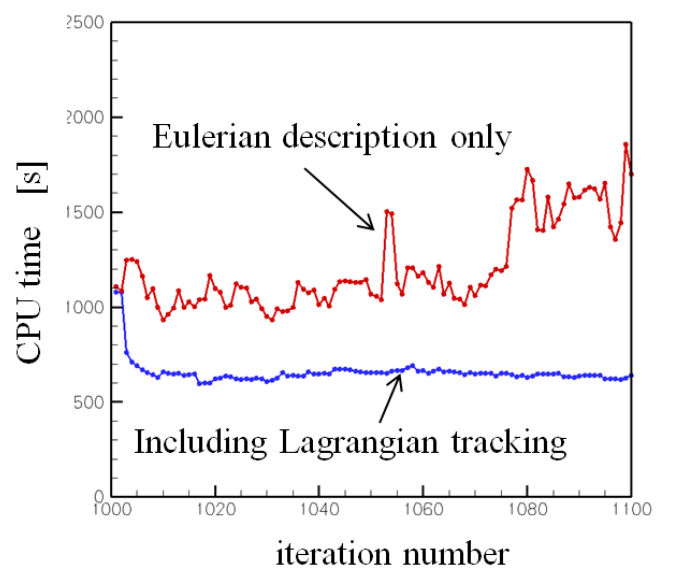

Figure 5. CPU time (in seconds) as a function of time step.

\section{Results}

\section{A. Liquid Jet in Crossflow}

The example chosen for demonstration comes from a series of tests carried out by Leong and Hautman ${ }^{19}$ for jet in crossflow, where droplet size, velocity and volumetric flux data were acquired using a Phase Doppler Particle Analyzer (PDPA). The measured properties of the test liquid (Jet-A) were density, $\rho_{l}=780 \mathrm{~kg} / \mathrm{m}^{3}$, surface tension $\sigma=0.024 \mathrm{~N} / \mathrm{m}$, and dynamic viscosity $\mu_{l}=0.0013 \mathrm{~kg} / \mathrm{m} / \mathrm{s}$. Air was at standard conditions, with inlet velocity of 69 $\mathrm{m} / \mathrm{s}$ (corresponding to a Weber number of 180 ). The orifice size was $d_{0}=0.762 \mathrm{~mm}$, with nominal mass flow rate $\dot{m}$ $=15.3 \mathrm{~kg} / \mathrm{h}$, and liquid jet Reynolds number of 3490. Although the experiment was guided by the attempt of measuring the spray characteristics as close as possible to injection, droplet sampling was still located as far as 33 orifice diameters downstream of the injector. This distance is dictated by the range of operation of the PDPA, which requires a dilute spray to provide accurate readings.

In the simulation, the coordinate system has the $x$-axis in the crossflow direction and the $z$-axis in the spanwise direction. The origin is located at the center of the jet orifice. The computational domain is a box of $5.12 \mathrm{~cm}$ in the $x$-direction $(1.28 \mathrm{~cm}$ upstream of the orifice) and of $2.56 \mathrm{~cm}$ in the $y$ and $z$ directions, see Figure 6 . The base resolution of $256 \times 128 \times 128$ nodes and 3 levels of refinement bring the minimum grid size to $\Delta x=25 \mu \mathrm{m}$.

The no-slip boundary condition is imposed at the $z=0$ plane, except at the jet orifice. Inlet boundary is imposed at the $x=-1.28 \mathrm{~mm}$ plane, and out-flow boundary condition are imposed on the remaining boundary planes. A plug flow profile is used for both the liquid and gas inlet. While trivial, this setting can be thought of as a preliminary study where the effect of inlet turbulence is excluded, leaving only to fluid mechanic instabilities the task of atomizing the jet. The droplet size factor is $m=4$, the drop critical sphericity factor is $\alpha_{c r i}=2$, and the critical density factor is $\beta_{c r i}=0.01$.

The simulation starts from the instant when the jet is injected into a well-developed crossflow gas $(t=0)$. The liquid jet penetrates into the gas flow, and, once the jet has reached full penetration, the computational methodology described in the previous Section limits the CPU time to 300-400 seconds per time step ( $\Delta t=0.17 \mu \mathrm{s})$ on two 8core, $32 \mathrm{~Gb}, 3000 \mathrm{MHz}$ nodes with InfiniBand switch.

The column is fragmented into ligaments/large drops in the tip of the jet, where large surface waves develop and the column experiences the strongest shear from the gas, see Figure 6. Large droplets further break up into smaller droplets downstream. Along the side of jet column, surface stripping of small droplets also occurs. Isolated liquid structures of substantially different size and shape co-exists at any instant of the process.

Figure 7 demonstrates the effects of grid-refinement on the formation of droplets and their subsequent transport. In frame (a), a base grid of $128 \times 64 \times 64$ with 3 level of refinement is used, compared to a base grid of $256 \times 128 \times 128$ with the same number of levels in frame (b). Although the large-scale jet penetration is little affected by grid resolution, the small-scale breakup details are quite different under the different resolutions. Particularly, the 
size of resulting droplets decreases with decreasing grid size. Since the volume loading is the same, the fine-grid simulation generates a larger number of droplets.

The aerodynamic blockage effect on the spatial distribution of droplets is illustrated by the front view of the jet in Figure 6 and by the top view in Figure 8a. The spatial distribution of droplets in the latter view compares qualitatively with tomograms of jet cross-sections ${ }^{24}$ in Figure $8 \mathrm{~b}$. In those pictures, most of the drops appear to be shed from the edges of the flattened jet. However, ligaments leaving the jet top follow a much shallower trajectory than in the experiment, because of the current lack of coupling of particles to the Eulerian representation.

A quantitative analysis is displayed in Figure 9, where the droplet size distribution from PDPA measurements at a plane 33 orifice diameters downstream of injection (dashed line in Figure 6b) is compared to the frequency distribution from the calculation at the higher grid density. A stationary spray distribution is assumed to occur at the sampling plane after $t=1.686 \mathrm{~ms}$ (the time of the snapshot in Figure 6). Particles are sampled until $t=1.884 \mathrm{~ms}$, for a total of 3470 drops. Both distributions in Figure 9 are normalized to unitary area. The data are concentrated between 10 and $50 \mu \mathrm{m}$, whereas the calculated droplets spread in a range between 30 and $120 \mu \mathrm{m}$. This is not completely unexpected, since the minimum $\Delta x$ of the simulation is only $25 \mu \mathrm{m}$. One, or possibly two, additional levels of refinement should be used to obtain a fully converged calculation. Work is in progress to achieve a minimum grid spacing of $12.5 \mu \mathrm{m}$, but the current results once again underscore the challenge posed to the modeling community by liquid atomization, particularly in the high-shear regime.

The comparison of the volumetric flux and size spatial distribution at the measurement plane is shown in Figure 10. Data are processed by interpolating the PDPA values (defined at the square locations) on a grid with half the grid spacing. The same procedure is followed for the simulation results, starting from discrete $0.635 \times 0.635 \mathrm{~mm}$ bins where the volumetric flux is not zero. The most substantial difference between experiment and calculation is the much reduced penetration and dispersion of the spray in the latter. This results in a more concentrated volumetric flow rate closer to the wall, as illustrated by Figure 10a compared to Figure 10b. This error can be alleviated by delaying the Lagrangian transformation further downstream of breakup, but we expect the problem to be removed altogether by implementing source terms from the Lagrangian particles in the Navier-Stokes momentum equation.

Even with the current limitations, the calculation captures the typical horseshoe flow rate distribution that is observed in LJIC atomization when the jet possesses sufficient penetration. It also qualitatively captures the Sauter mean drop (SMD) distribution, with larger drops drifting at the periphery of the spray and smaller particles in the wake of the jet. The calculated size distribution, from 40 to $90 \mu \mathrm{m}$, is shifted toward larger drops by approximately $20 \mu \mathrm{m}$, consistent with the overall size distribution of Figure 9.

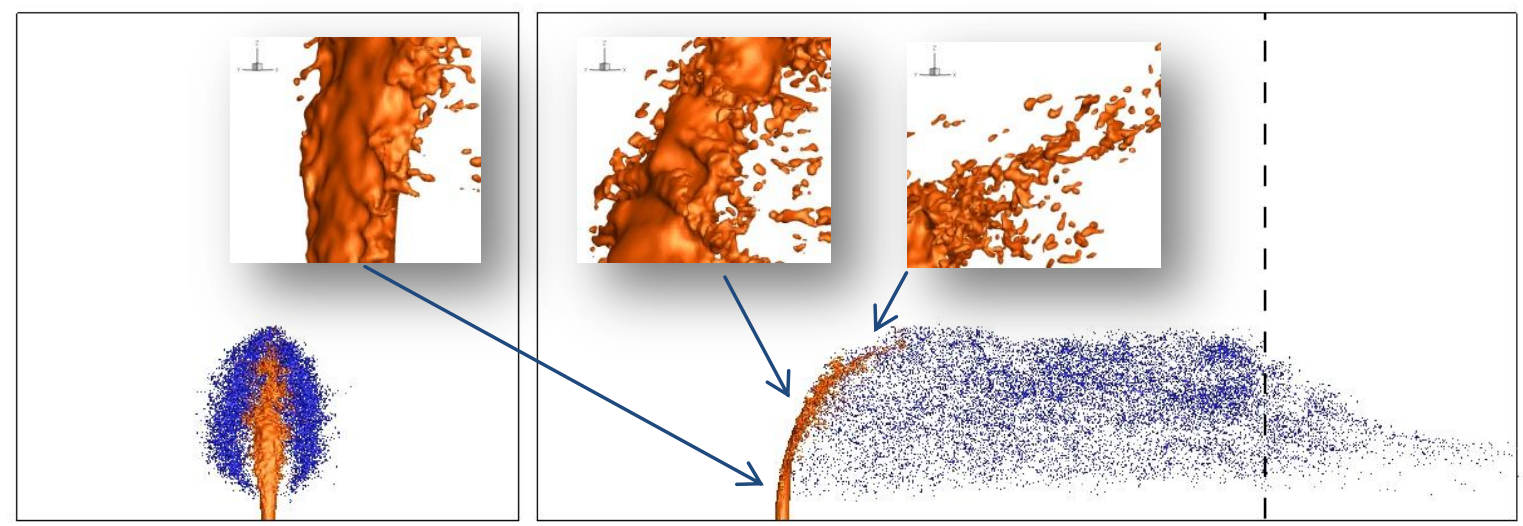

Figure 6. Front (a) and side view (b) of the jet at $t=1.686 \mathrm{~ms}$. The jet calculated by CLSVOF on the Eulerian grid is represented by the rendering of the 0 level set iso-surface. The spray (Lagrangian) is shown as a scatter of spheres whose diameter is scaled to the plot. The continuous lines delimit the boundary domain, the dashed line is the trace of the sampling plane. Different segments of the iso-surface only, from near injection to breakup, are also displayed in the three inserts. 


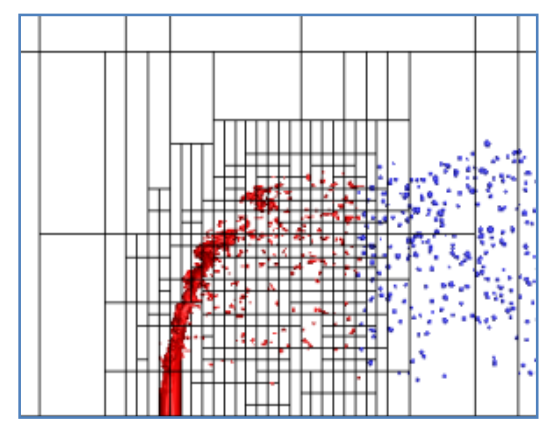

(a)

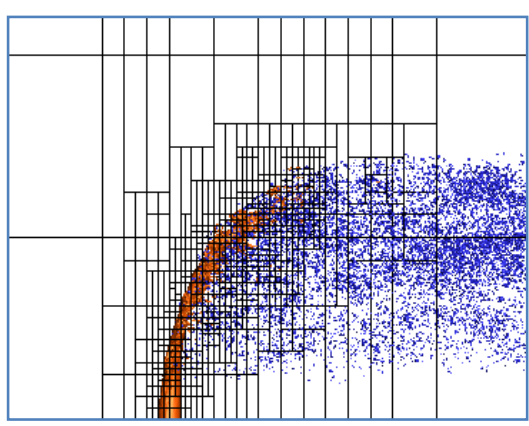

(b)

Figure 7. JICF simulation side view after 6000 time steps: coarse grid (a) and fine grid (b).

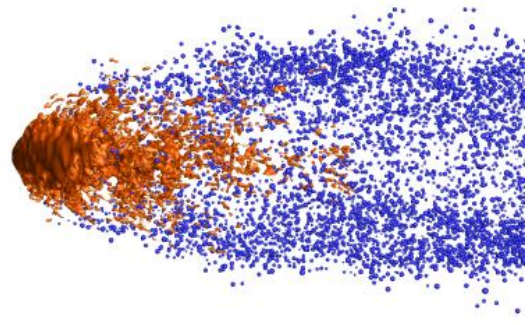

(a)

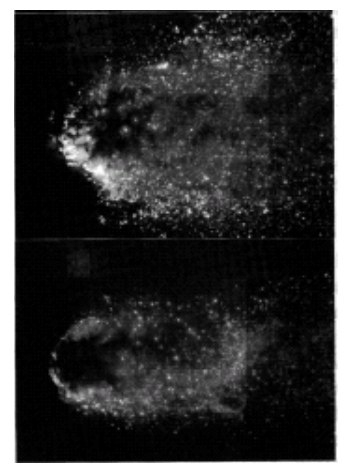

(b)

Figure 8. Fine grid calculation top view (a) and two cross-sectional tomograms by Oda et al. ${ }^{24}$ displayed for qualitative comparison (b).

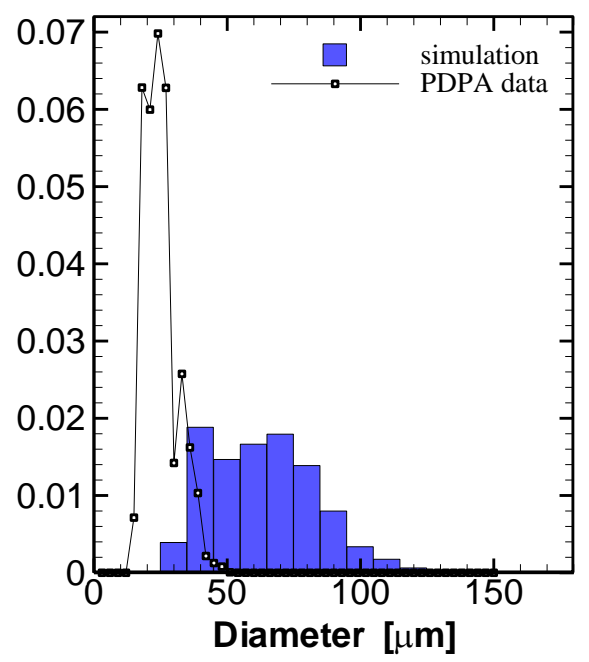

Figure 9. Droplet size distribution from PDPA (symbols) and from simulation. 


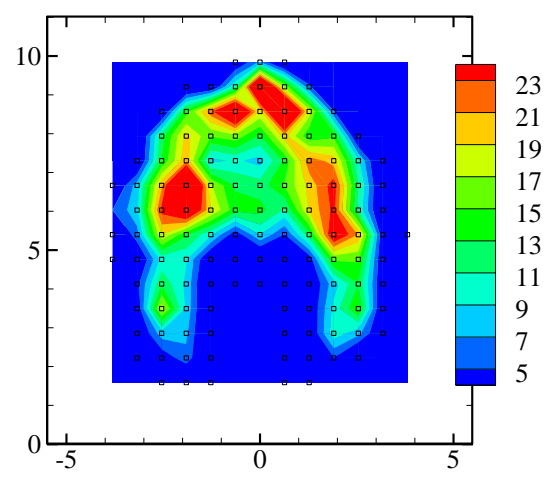

(a)

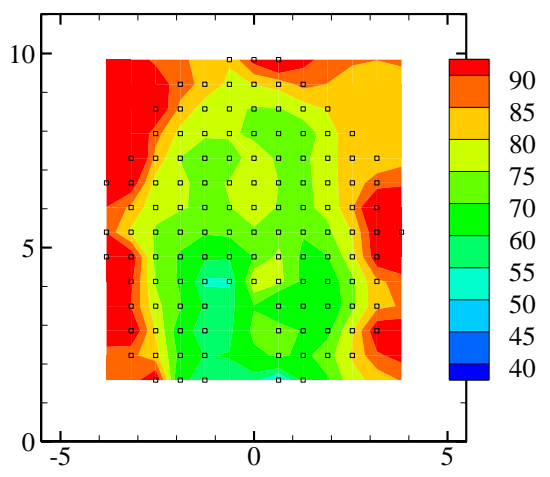

(c)

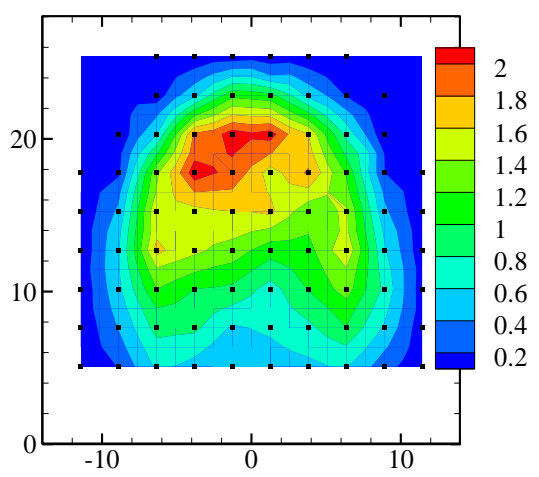

(b)

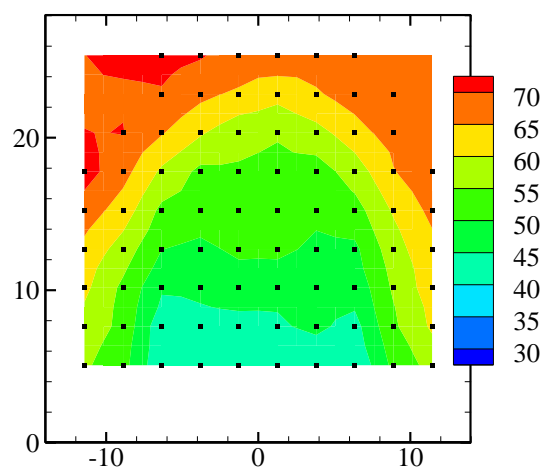

(d)

Figure 10. Spray distribution at $x=33$ orifice diameters downstream of injection: volumetric flow rate (in $\mathrm{cc} / \mathrm{cm}^{2} / \mathrm{s}$ ) from the simulation (a) and PDPA measurement (b); Sauter Mean Diameter (in $\mu \mathrm{m}$ ) from the simulation (c) and the measurement (d).

\section{B. Impinging Jets}

The calculation presented here simulates a simple experiment where two water jets are injected into quiescent air at atmospheric pressure and collide with each other to form a liquid sheet. This case is taken from a series of likeon-like jet impingement experiments ${ }^{20,21}$ that report phase Doppler particle analyzer (PDPA) measurements at the spray fan centerline. In the experiment, precision bore glass tubes with internal diameter $d_{0}=0.635 \mathrm{~mm}$ and lengthto-diameter ratio of 80 were oriented to generate an impingement angle of $60^{\circ}$ and a pre-impingement length of $l_{j}=$ $25.4 \mathrm{~mm}$. Drop size measurements were taken $16 \mathrm{~mm}$ downstream of the impingement point, using approximately 8000 samples to achieve $\pm 5 \%$ accuracy in mean diameter. The PDPA optical configuration was set to provide a 40 to $1400 \mu \mathrm{m}$ range of drop diameters. The test liquid was water $\left(\rho_{l}=998 \mathrm{~kg} / \mathrm{m}^{3}\right.$, surface tension $\sigma=0.076 \mathrm{~N} / \mathrm{m}$, and dynamic viscosity $\mu_{l}=0.0010 \mathrm{~kg} / \mathrm{m} / \mathrm{s}$ ).

A snapshot of the simulation is shown in Figure 11, including the rendered level set 0 iso-surface from CLSVOF and the Lagrangian particles (in lighter color). Injection occurs in the $y-z$ plane, with the $z$ axis aligned in the common direction of the two jets. To reduce the overall size of the computational domain, the bottom plane (at $z$ $=0$ ) is not the injection wall of the experiment, but rather truncates the two liquid columns at the pre-impingement length $l_{j}=4 \mathrm{~mm}$. Inflow boundary conditions are set for the gas phase outside the two orifices on that plane, outflow boundary conditions are enforced on all the other planes. Since the liquid velocity is a plug flow profile, as in the liquid jet in crossflow calculation, no attempt is made to mimic wall effects, or inner turbulence effects, or the relaxation of the velocity profile after injection - the exclusion of these effects allows us to concentrate on the fluid dynamics of jet impingement. The overall box size is 16 by 12 by $24 \mathrm{~mm}$, leaving sufficient room to sample droplets 
at $z=20 \mathrm{~mm}$. With one exception, the results presented here refer to a calculation with 2 levels of refinement, leading to a minimum grid size of $\Delta \mathrm{x}=31.25 \mu \mathrm{m}$ slightly below the PDPA minimum range. The parameters governing the transformation to the Lagrangian description are $m=4, \alpha_{c r i}=2, \beta_{c r i}=0.01$.

Two snapshots with and without Lagrangian drops (Figure 12a and b) illustrate the blend of the two representations of the liquid phase. In the continuous representation, waves can be observed to form downstream of jet impingement, and tears along the intact sheet deepen until they detach thin ligaments from the periphery of the sheet. The rendered zero level set iso-surface in the figure does not provide a sense for the thickness of the sheet, but further inspection and the comparison with snapshots from the experiment ${ }^{20}$ reveal the existence of very thin membranes, where breakup initiates, and of thicker crests between them. The ligaments fracture rather quickly and their fragments equilibrate into almost spherical droplets that carry the residual momentum of the jet. Since the air is quiescent, the lack of two-way momentum coupling has a more limited impact than in the jet in crossflow case. While the ligaments display some regular spacing near the core, the droplets disperse in an apparently more disorganized pattern in the far field. Further analysis is necessary to establish whether the spray flow rate exhibits any natural coherence.

The spray diameter distribution at the $z=20 \mathrm{~mm}$ plane is compared with the PDPA measurements in Figure 13. For completeness, frame (a) displays the distribution from a preliminary calculation with only one level of refinement, or minimum grid spacing of $62.5 \mu \mathrm{m}$. The impinging sheet for that simulation lacks several of the fine structures of Figure 11, see the comparison in Figure 14. The droplets resulting from the coarsest simulation are substantially larger, with a peak occurrence at $200 \mu \mathrm{m}$. The distribution with two levels of refinement in frame Figure 13b, with a peak at approximately $100 \mu \mathrm{m}$, demonstrates instead a remarkable agreement with the data. Droplets were sampled between 5.24 and $6.58 \mathrm{~ms}$ to exclude initial startup effects, for a total of 1086 samples. In this interval, the CPU time was approximately 150 seconds per time step ( $\Delta t=0.66 \mu \mathrm{s}$ ) on two 8-core, $32 \mathrm{~Gb}, 3000$ $\mathrm{MHz}$ nodes with InfiniBand switch

This Section is concluded by a study on mass conservation of the overall methodology. The mass flow rate through the two orifices is kept constant during the simulation and therefore the total liquid mass that gets introduced in the computational domain is linear, until droplets begin to exit the domain. In the time interval displayed on Figure 15, the amount of liquid volume represented by CLSVOF on the AMR grid has reached a stationary value, since the input mass is on average balanced by the mass of newly created Lagrangian drops. This plot corresponds to the sequence of triangles on Figure 15. The volume of all the droplets contained in the domain, shown by the sequence of square symbols, begins to approach a stationary value by $t=5.5 \mathrm{~ms}$. Finally, the volume of all the droplets that have left the domain is shown by the diamond symbols. If these three components are summed at each time step, the result is an approximately straight line (circle symbols), with oscillations that are less than $1 \%$ of the mass value taken at $t=2.5 \mathrm{~ms}$. This result proves the excellent convergence properties of CLSVOF, as well as the correct implementation of the Eulerian to Lagrangian transition algorithm. 


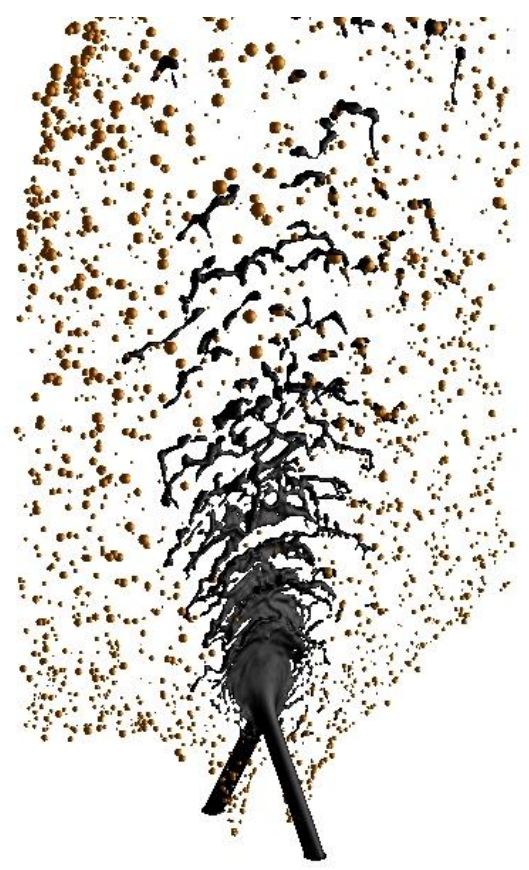

Figure 11. Isometric view of the impinging jets at $t=7.659 \mathrm{~ms}$.

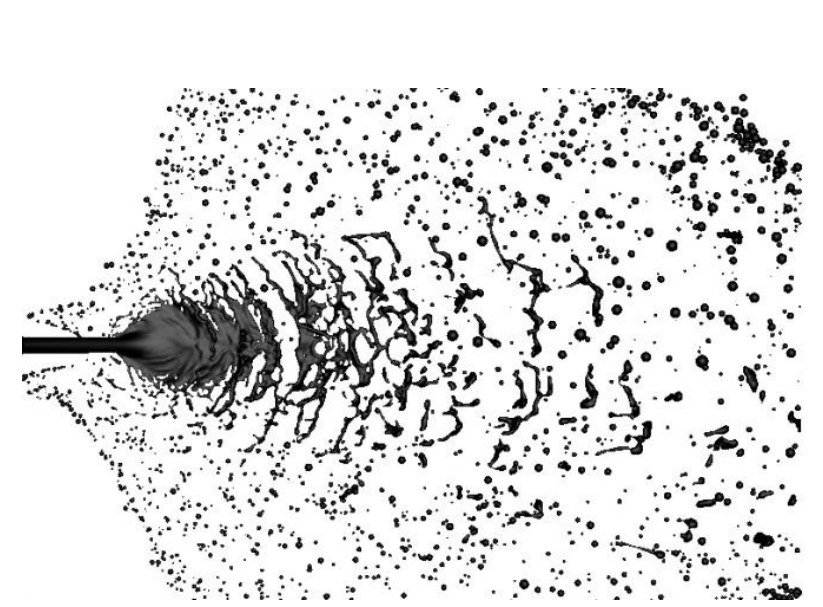

(a)

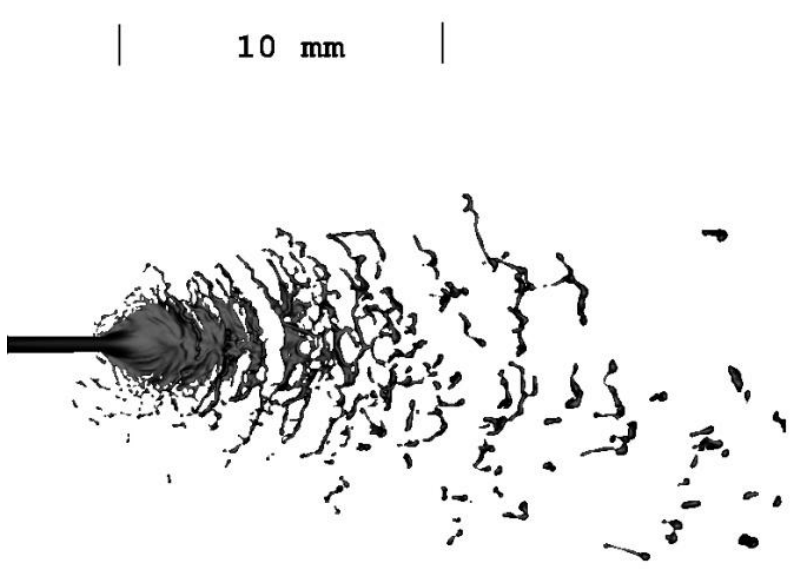

(b)

Figure 12. Side view of the impinging jets at $t=6.54 \mathrm{~ms}$ : (a) Complete simulation; (b) Eulerian discretization only. 


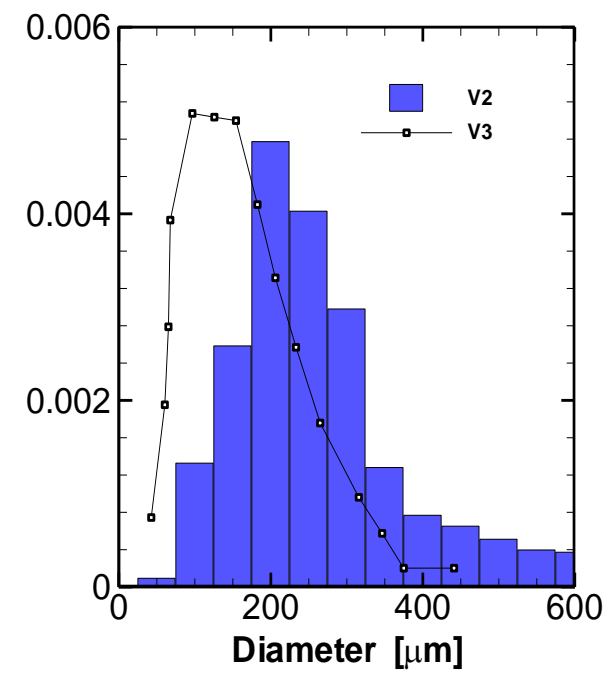

(a)

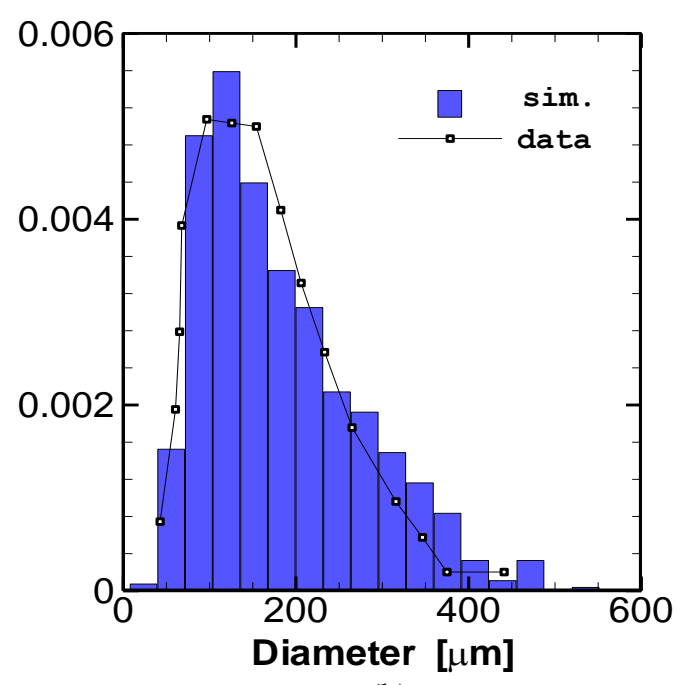

(b)

Figure 13. Comparison of the density distribution of droplet size from experiment ${ }^{21}$ (symbols) and from the simulation: (a) 1 level of refinement with minimum $\Delta x=62.5 \mu \mathrm{m}$; (b) 2 levels of refinement with minimum $\Delta x$ $=\mathbf{3 1 . 2 5} \mu \mathrm{m}$.

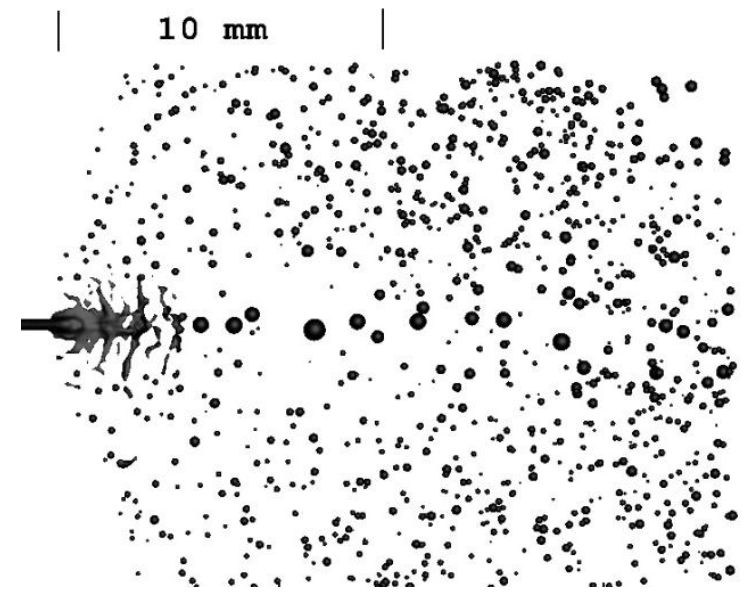

(a)

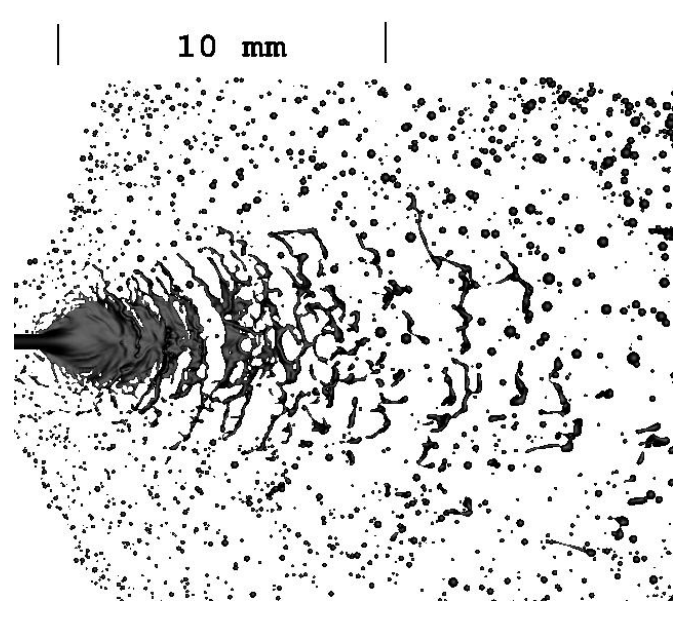

(b)

Figure 14. Two side view snapshots of the simulation: (a) 1 level of refinement with minimum $\Delta x=62.5 \mu \mathrm{m}$; (b) 2 levels of refinement with minimum $\Delta x=31.25 \mu \mathrm{m}$. 


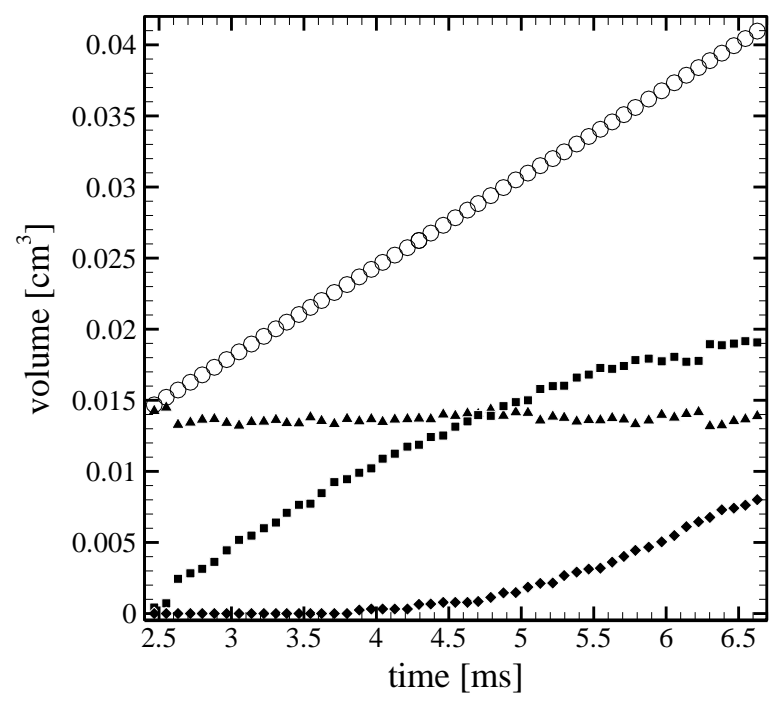

Figure 15. Liquid volume variation as a function of time: $(\Delta)$ volume in the Eulerian representation; (b) ( $(\square)$ volume in the Lagrangian droplet inside the computational domain: ( $\downarrow)$ volume in the droplets that have left the domain; ( $\circ$ ) sum of all the previous terms.

\section{Summary}

The contribution of the present work is to show how a combination of adaptive mesh refinement and Lagrangian tracking can enable high-fidelity simulations of liquid jet atomization and of spray dispersion. Jet penetration, fluid dynamic interaction with the gas, and eventual breakup of the liquid are simulated directly using the CLSVOF method embedded in block-structured dynamic mesh refinement. The micro-scale of dilute spray droplets are transformed into Lagrangian particles and transported using discrete phase models. We have shown that using appropriate droplet identification criteria and a simple transport model, the Lagrangian tracking of droplets produces accurate results compared to the Eulerian-only simulation. The transformation effectively reduces the calculation cost by alleviating the mesh refinement required to resolve micro-scale droplets, therefore localizing mesh refinement very near the orifice. It is now feasible to compare computed spray characteristics with data in a realistic simulation of the experiment. Preliminary results for two demonstration cases compare favorably with the measured spray characteristics and support the feasibility of the proposed approach. The demonstration of full grid convergence and the addition of momentum and energy coupling to the Navier-Stokes equation will be the immediate next steps in this work.

\section{Acknowledgements}

The authors wish to acknowledge the guidance of Dr. R. Jensen (PWR in Canoga Park, CA) and the support of United Technologies Corp. They also thank Chris Eckett (UTRC) and Sophia Lefantzi (UTRC) for their contribution. One of the authors (Sussman) was partially supported by the NSF grant DMS 0713256. The code performance optimization that enabled this work used resources of the National Energy Research Scientific Computing Center, which is supported by the Office of Science of the U.S. Department of Energy under Contract No. DE-AC02-05CH11231. Particularly, the expertise and time commitment of Viraj Paropkari and David Skinner from the SciDAC Outreach Center are gratefully acknowledged for their impact on the project objectives. 


\section{References}

1. Sethian, J. A., Smereka, P., "Level Set Methods for Fluid Interfaces," Annual Review of Fluid Mechanics 35: 341-372, 2003.

2. Scardovelli, R., Zaleski, S., Direct Numerical Simulation of Free-Surface and Interfacial Flow," Annual Review of Fluid Mechanics 31: 567-603, 1999.

3. Tryggvason, G., Bunner, B., Esmaeeli, A., Juric, D., Al-Rawahi, N., Tauber, W., Han, J., Nas, S., and Jan, Y. J., "A fronttracking method for the computations of multiphase flow," Journal of Computational Physics 169(2): 708-759, 2001.

4. Herrmann, M., "A balanced force refined level set grid method for two-phase flows on unstructured flow solver grids," Journal of Computational Physics, 227(4) 2674-2706, 2008.

5. Himeno, T., Watanabe, T., and Konno, A., "Numerical analysis for propellant management in rocket tanks," Journal of Propulsion and Power, 21 (1) 76-86, 2005.

6. Sussman, M., Smith, K. M., Hussaini, M.Y., Ohta, M., and Zhi-Wei, R., "A sharp interface method for incompressible twophase flows," Journal of computational physics, 221 469-505, 2007.

7. Černe, G., Petelin S., and Tiselj, I, "Numerical errors of the volume-of-fluid interface tracking algorithm," Int. J. Numer. Meth. Fluids 38 329-350, 2002.

8. Herrmann, M., "A parallel Eulerian interface tracking/Lagrangian point particle multi-scale coupling procedure,” Journal of Computational Physics, 229(3) 745-759, 2010.

9. Herrmann, M., Proceedings of ASME Turbo Expo, Power for Land, Sea \& Air, 59563, Orlando, FL, 2009.

10. Salman, H. and Soteriou, M., "Lagrangian Simulation of Evaporating Droplet Sprays," Physics of Fluids, 16(12) 4601-4622, 2004.

11. R. H. Rangel and W. A. Sirignano, “An Evaluation of the Point-Source Approximation in Spray Calculations,” Numerical Heat Transfer, Part A 16, 37 (1989).

12. M. J. Berger and P. Colella, "Local adaptive mesh refinement for shock hydrodynamics," Journal of Computational Physics, 82(1) 64-84, 1989.

13. Kadioglu, S.Y. and Sussman, M., "Adaptive solution techniques for simulating underwater explosions and implosions," Journal of Computational Physics 227 2083-2104, 2008.

14. Almgren, A.S., Bell, J.B., Colella, P., Howell, L.H., and Welcome, M. L., "A conservative adaptive projection method for the variable density incompressible Navier-Stokes equations, Journal of Computational Physics, 142 1-46, 1998.

15. Wu, P. K., Kirkendall, K. A., Fuller, R. P., and Nejad, A. S., "Break-up processes of liquid jets in subsonic crossflows", Journal of Propulsion and Power, 13 64-73, 1997.

16. Becker, J., and Hassa, C., "Breakup and atomization of a kerosene jet in crossflow at elevated pressure," Atomization and Sprays, 11 49-67, 2002.

17. Arienti, M., Madabhushi, R. K., Van Slooten, P. R., and Soteriou, M. C., Proceedings of ASME Turbo Expo, Power for Land, Sea \& Air, 90536, Barcelona, Spain 2006.

18. Pai, M. G., Pitsch, H., and Desjardins, O., "Detailed numerical simulations of primary atomization of liquid jets in crossflow," 47 $7^{\text {th }}$ AIAA Aerospace Sciences Meeting, Orlando, FL., 2009, AIAA 2009-0373.

19. Hautman, D. J., "Spray characterization of like-on-like doublet impinging rocket injectors," $27^{\text {th }}$ AIAA ASME SAE ASEE Joint Propulsion Conference, AIAA 1991-0687.

20. Ryan, H. M., Anderson, W. E., Pal, S., and Santoro, R. J., “Atomization characteristics of impinging liquid jets,” Journal of Propulsion and Power, 11(1) 135-145, 1995.

21. Anderson, W. E., Ryan, H. M., Santoro, R. J., and Hewitt, R. E., "Combustion instability mechanism in liquid rocket engines using impinging jet injectors, $31^{s t}$ AIAA ASME SAE ASEE Joint Propulsion Conference, AIAA 95-2357.

22. Liu, A.B., Mather, D., and Reitz, R.D., "Modeling the effects of drop drag and breakup on fuel sprays", SAE Technical Paper 930072, SAE, 1993.

23. Leong, M. Y., Hautman, D. J., ILASS Americas, $15^{\text {th }}$ Annual Conference on Liquid Atomization and Spray Systems, Madison, WI, May 2002.

24. Oda, T., Nishida, K., Hiroyasu, H., Sixth International Conference on Liquid Atomization and Spray Systems, pp. 624-631, Rouen, France, July 1994. 